\title{
A 2D modeling approach for fluid propagation during FE-forming simulation of continuously reinforced composites in wet compression moulding
}

Christian Poppe, Dominik Dörr, Frank Henning, and Luise Kärger

Citation: AIP Conference Proceedings 1960, 020022 (2018); doi: 10.1063/1.5034823

View online: https://doi.org/10.1063/1.5034823

View Table of Contents: http://aip.scitation.org/toc/apc/1960/1

Published by the American Institute of Physics

\section{Articles you may be interested in}

A cyber physical system approach for composite part: From smart manufacturing to predictive maintenance AIP Conference Proceedings 1960, 020025 (2018); 10.1063/1.5034826

Forming of complex-shaped composite tubes using optimized bladder-assisted resin transfer molding AIP Conference Proceedings 1960, 020027 (2018); 10.1063/1.5034828

Multi-scale modelling of non-uniform consolidation of uncured toughened unidirectional prepregs AIP Conference Proceedings 1960, 020032 (2018); 10.1063/1.5034833

Comparison of validation methods for forming simulations AIP Conference Proceedings 1960, 020028 (2018); 10.1063/1.5034829

A meta-model based approach for rapid formability estimation of continuous fibre reinforced components AIP Conference Proceedings 1960, 020042 (2018); 10.1063/1.5034843

Prediction of composites behavior undergoing an ATP process through data-mining AIP Conference Proceedings 1960, 020016 (2018); 10.1063/1.5034817 


\title{
A 2D modeling approach for fluid propagation during FE- forming simulation of continuously reinforced composites in wet compression moulding
}

\author{
Christian Poppe ${ }^{1 a}$, Dominik Dörr ${ }^{1}$, Frank Henning ${ }^{1,2}$, Luise Kärger ${ }^{1}$ \\ ${ }^{1}$ Karlsruhe Institute of Technology, Institute for Vehicle System Technology, Chair of Light-Weight Technology, \\ Rintheimer Querallee 2, 76131 Karlsruhe, Germany \\ ${ }^{2}$ Fraunhofer Institute for Chemical Technology, Polymer Engineering Department, Joseph-von-Fraunhofer-Str. 7,
} 76327 Pfinztal, Germany

a) Corresponding author: christian.poppe@kit.edu

Keywords: FE-Forming Simulation, FE/CV, Wet compression moulding (WCM), mould filling, FSI

\begin{abstract}
Wet compression moulding (WCM) provides large-scale production potential for continuously fiber reinforced components as a promising alternative to resin transfer moulding (RTM). Lower cycle times are possible due to parallelization of the process steps draping, infiltration and curing during moulding (viscous draping). Experimental and theoretical investigations indicate a strong mutual dependency between the physical mechanisms, which occur during draping and mould filling (fluidstructure-interaction). Thus, key process parameters, like fiber orientation, fiber volume fraction, cavity pressure and the amount and viscosity of the resin are physically coupled. To enable time and cost efficient product and process development throughout all design stages, accurate process simulation tools are desirable. Separated draping and mould filling simulation models, as appropriate for the sequential RTM-process, cannot be applied for the WCM process due to the above outlined physical couplings. Within this study, a two-dimensional Darcy-Propagation-Element (DPE-2D) based on a finite element formulation with additional control volumes $(\mathrm{FE} / \mathrm{CV})$ is presented, verified and applied to forming simulation of a generic geometry, as a first step towards a fluid-structure-interaction model taking into account simultaneous resin infiltration and draping. The model is implemented in the commercial FE-Solver Abaqus by means of several user subroutines considering simultaneous draping and 2D-infiltration mechanisms. Darcy's equation is solved with respect to a local fiber orientation. Furthermore, the material model can access the local fluid domain properties to update the mechanical forming material parameter, which enables further investigations on the coupled physical mechanisms.
\end{abstract}

\section{INTRODUCTION}

Wet compression moulding (WCM) provides large-scale production potential including low cycle times due to the parallelization of the process steps draping, infiltration and curing during moulding (cf. Fig. 1).

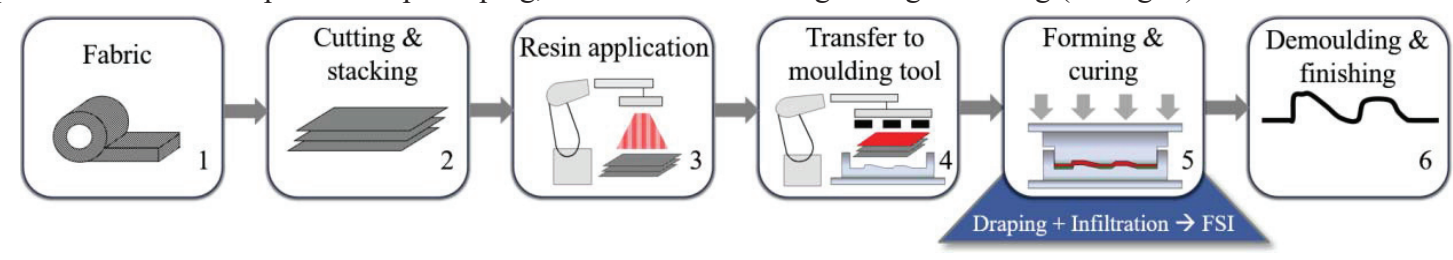

FIGURE 1. Illustration of the WCM-Process steps with additional emphasis on the crucial simulations of draping and mould filling (viscous draping) in step (5).

According to Bergmann et al. [1,2] the final part's shape and performance produced with the WCM technology depends on several key process parameters: stack weight and storing duration, resin amount, component temperatures, 
infiltration time, pressing force/maximum pressure, sequence and tool temperatures. Moreover, experimental and theoretical investigations indicate a strong mutual dependency between the physical mechanisms within the viscous draping-stage (cf. Fig. 1 (5)). However, it is still unknown which mutual dependency arise at which point in time during the tool stroke and how these dependencies influence the local structural properties of the final part. Thus, an accurate, physical-based process simulation model is required, which is capable to simultaneously describe a multilayer draping process and the resin propagation within the mould. Existing sequential draping and mould filling simulations models that are appropriate for the RTM-process, cannot be applied to the WCM process due to the above outlined physical couplings. While forming simulation models utilize Lagrangian meshes in combination with the Finite Element Method (FEM) to describe the deformation behaviour of a solid body, mould-filling simulations usually are based on Eulerian meshes to describe the fluid dynamics using e.g. the Finite-Volume-Method (FVM). Beyond that, mould filling with single-phase porous media flows can alternatively be modelled using Finite Elements with a Galerkin formulation, supplemented by additional control volumes (FE/CV) to ensure mass conservancy, as presented by Trochu et al. [4] and Bruschke et al. [5].

In this work, a new two-dimensional Darcy-Propagation-Element (DPE-2D) is presented, based on an FE/CV approach as a first step towards a physical-based process simulation model for the WCM-process. For this purpose, the resin propagation within a single layer of the laminate is constitutively described simultaneously to the forming of the laminate. The presented approach is implemented within a user-element subroutine of the commercial FE-solver Abaqus. The new element is verified by means of one- and two-dimensional mould filling test scenarios by comparison with analytical and numerical solutions $\left(\mathrm{PAM}-\mathrm{RTM}^{\circledR}\right)$ and finally applied to forming of a generic geometry.

\section{NUMERICAL APPROACH}

As motivated in the previous section, an FEM approach on a macroscopic scale is used for numerical process simulation. The framework for the new element is based on an forming simulation approach for thermoplastic CFRPs presented by Dörr et al. [7,8], which is implemented in several user-subroutines of the commercially available FE solver Abaqus. In general, FE forming simulation bases on constitutive modeling of the relevant deformation mechanisms during forming, which are usually categorized according to intra-ply and interface mechanisms. To account for interface mechanisms, which are the mechanisms between the single plies of the stacked laminate (cf. Fig. 2 (b)), as well as between the tool and the laminate, each ply is modelled by separate element layers. Each element layer consists of a membrane and a shell element, and is additionally superimposed by a Darcy-Propagation-Element, which does not contribute to the mechanical deformation by means of nodal forces yet (cf. Fig. 2 (c)), but takes deformation and local fiber reorientation into account while solving the pressure field.

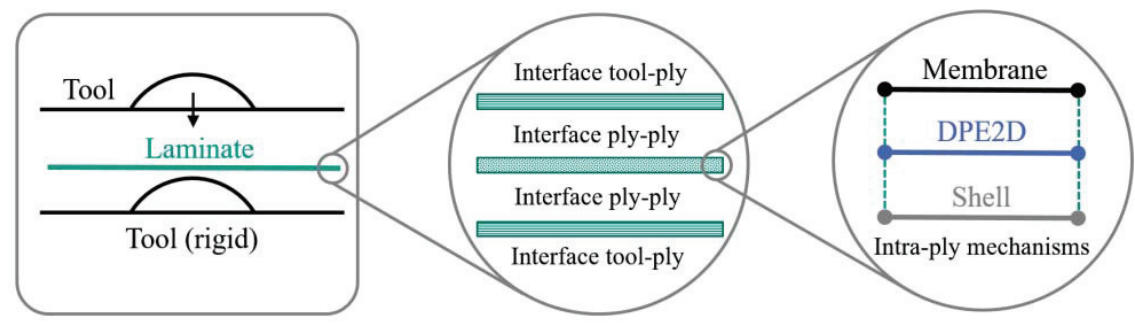

(a) Overall model setup

(b) Stacked single plies

(c) Internal ply structure

FIGURE 2. Schematic illustration of the FE-model setup in Abaqus comprising stacked single plies consisting of three superimposed and node-sharing elements to account for the intra-ply mechanisms.

The separate element-layers of each single ply are capable of modeling membrane and bending behaviour (intraply mechanisms) in a decoupled fashion, which is crucial for forming simulation of continuously reinforced [8]. An explicit time integration scheme is used due to the large contact areas within forming simulation.

\section{Darcy Propagation Element (DPE-2D)}

Two mechanisms need to be implemented for the new element, on the one hand the solution of the pressure field within the saturated domain using Darcy's law and on the other hand the corresponding propagation of the resin front by utilizing additional control volumes (CVs). Every element is assigned a saturation value, which determines its 
behaviour during the analysis. Due to the constant fluid propagation, an element can be either within the actual saturated domain or outside. A saturated element contributes to the global solution of the pressure field with respect to the local fiber orientation. Empty or partially saturated elements gather incoming volume fluxes from saturated neighbouring elements (cf. Fig. 3 (a)). Beyond that, each element comprises three nodes connected through three edges and one integration point (cf. Fig. 3 (b)). The two mechanisms are presented in the following.

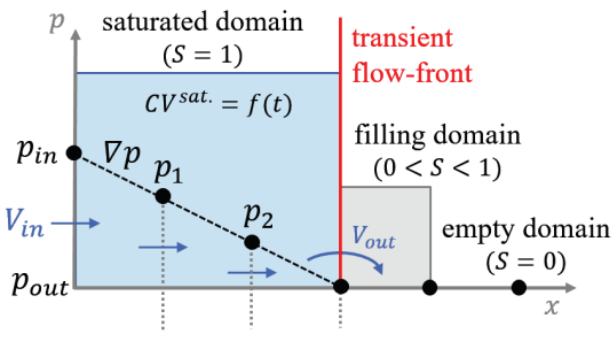

(a)

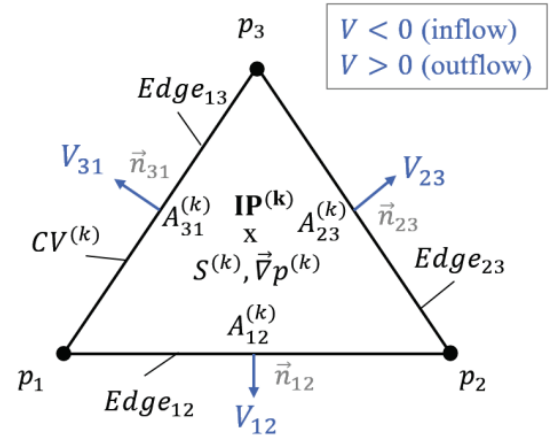

(b)

FIGURE 3. (a) Volume flux and Control Volumes for a 1D-flow example, (b) DPE-2D Element conventions.

Saturated domain. The flow of resin through a fibre bed can be assumed to be equivalent to a flow of an incompressible, isothermal fluid through a porous medium [9]. Hence, the two-dimensional pressure distribution $p(x, y)$ within a saturated domain can be described using Darcy's law

$$
\left(\begin{array}{l}
u_{x} \\
u_{y}
\end{array}\right)=-\frac{1}{\eta * \phi}\left(\begin{array}{ll}
K_{x x} & K_{x y} \\
K_{y x} & K_{y y}
\end{array}\right)\left(\begin{array}{l}
\partial P / \partial x \\
\partial P / \partial y
\end{array}\right)=-\frac{\boldsymbol{K}}{\eta * \phi} \operatorname{grad}(p)
$$

with additional respect to mass conservation

$$
\dot{V}=\int_{V}-\operatorname{div}(\vec{u}) \mathrm{d} V \rightarrow 0 .
$$

The system equation within the saturated domain is obtained by combination of equation (1) and (2)

$$
\dot{V}=\int_{V} \operatorname{div}\left(\operatorname{grad}\left(\frac{\boldsymbol{K}}{\eta * \phi} p\right)\right) \mathrm{d} V \rightarrow 0 .
$$

Here, $\eta$ is the fluid viscosity, $\boldsymbol{K}$ is the permeability tensor, $\phi$ the fiber volume content, $u_{x}, u_{y}$ the pressure corresponding fluid velocities to the nodal pressure $p$ and $\dot{V}$ the fluid volume rate within the saturated domain. Abaqus/explicit offers a degree of freedom (DoF) for pressure and temperature at its nodes, which can be accessed via the user subroutine (VUEL). In this work the temperature DoF is used to solve the pressure field within a fully coupled structural-thermal analysis, because of its accessibility within other built-in methods (e. g. contact). The use of the temperature DoF is possible due to the analogy of the equations for temperature (Fourier's Law) and pressure (Darcy's law with additional mass conservation (eq. 3)). In this manner, a similar description for Equation 3 as for transient thermal analyses is obtained by taking into account the following two analogies whereby $\lambda$ is the thermal conductivity and $c_{p}$ the specific heat capacity:

$$
\frac{K}{\eta} \sim \lambda \quad \& \quad C \sim \int_{V} c_{p} \rho \mathrm{d} V .
$$

Accordingly the hydraulic capacity $(C=\dot{V} / \dot{P})$ is introduced by

$$
\int_{V} c_{p} \rho \frac{\partial T}{\partial t} \mathrm{~d} V=\int_{V}-\lambda * \operatorname{div}(\operatorname{grad}(T)) \mathrm{d} V \triangleq C \dot{P}=\int_{V} \frac{K}{\eta * \phi} \operatorname{div}(\operatorname{grad}(P)) \mathrm{d} V,
$$

whereby the hydraulic capacity $C$ should be as small as possible for representation of a quasi-incompressible fluid. 
Due to the usage of an explicit time-integration scheme, a complete incompressibility $(C=0)$ cannot be modeled, however numerical investigations reveal a sufficient range where deviations are negligible. The presented new element is implemented in a user element subroutine (VUEL) in Abaqus/explicit by utilizing the temperature DoF with respect to the above outlined analogies.

Flow front progression. Beyond the determination of the pressure distribution in the saturated domain, a physical based implementation of the flow front propagation within the single ply is needed, to ensure mass conservation. Therefore, a transient global control volume (CV) is implemented including all saturated elements (cf. Fig. 3 (a)). The two tasks of the global CV are the determination of the summed propagated flow at the flow front and its partition on the filling elements. The volume flow at every edge for saturated elements is calculated using the scalar product:

$$
V_{i}^{\mathrm{N}+1(k)}=\Delta t_{\mathrm{inc}} * A_{i}^{(k)}\left(\vec{n}^{(k)} * \vec{u}^{(k)}\right),
$$

where $V_{i}^{\mathrm{N}+1}$ is the new incremental volume flow of element $\mathrm{k}$ with respect to the actual edge $i$ according to the conventions in Fig. 3 (b). Since the summed incremental volume flow of every element is zero, mass conservation within every element is always implied. However, by comparing the calculated volume flow on both sides of the same edge, discrepancies observed by various researchers and explained by Josh et. al. [6] could be confirmed. Therefore, only outer edges of the global CV are considered in the summation of the propagation flow. Their contribution depends on their status (inlet, outlet, wall, flow front) to account for possible boundary conditions. Further, an algorithm based on the pressure gradient and the area of the filling elements is implemented to determine the partition of the summed propagation flow. The saturation values within the filling elements are updated accordingly until their saturation reaches one. This workflow is repeated incrementally within the analysis.

\section{VERIFICATION AND SUPERIMPOSED FORMING}

Within the scope of a verification, two scenarios without superimposed deformation are applied to the new element. The solution is compared to the analytical result and to the commercial mould filling simulation tool PAM-RTM ${ }^{\mathbb{R}}$ (Version 14.5). Afterwards a mould filling with superimposed forming of a double dome geometry is presented.

One-dimensional flow scenario. A strip of 1000 structured elements is filled with a one-dimensional flow under constant pressure (cf. Fig. 4 (a)). The pressure of 0,5 $\mathrm{MPa}\left(p_{\text {in }}\right)$ is predefined the nodes of the left side of the strip while the pressure at nodes at the right site stays $0 \mathrm{MPa}\left(p_{\text {out }}\right)$.

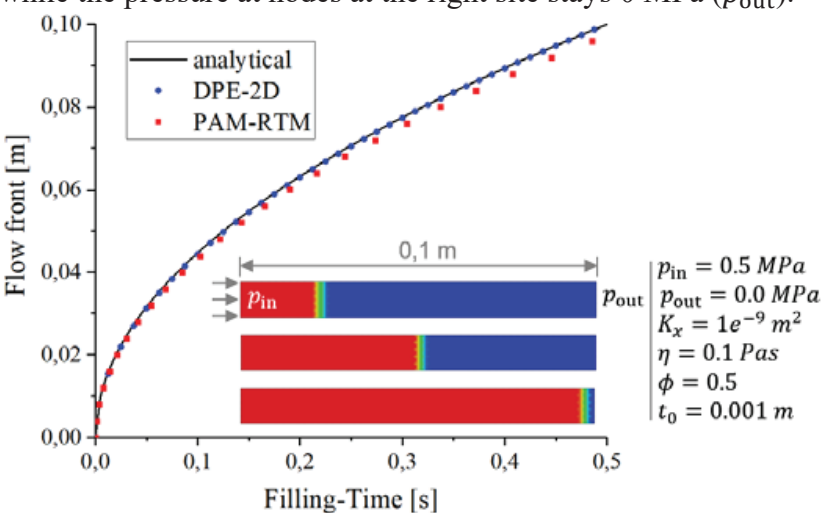

(a)

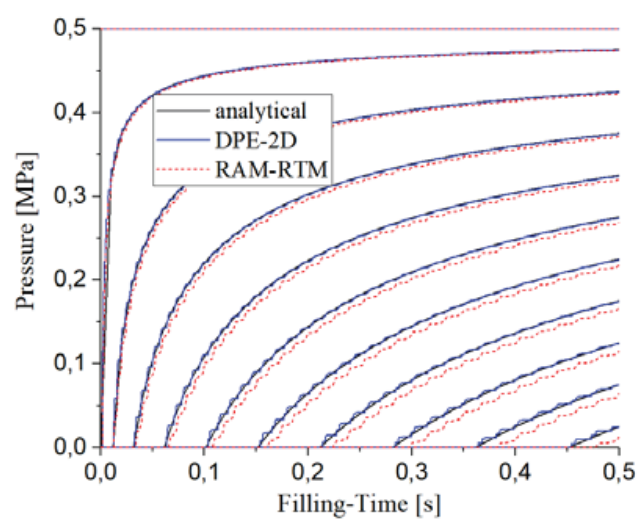

(c)

FIGURE 4. Verification results of the one-dimensional flow; (a) Progression of the flow front; (b) material parameters used; (c) Pressure propagation at equidistant nodes within a centerline of the test strip.

The results for the one-dimensional flow test case demonstrate a very good agreement to the analytical solution; this applies to the propagation of the flow front (cf. Fig. 4 (a)) as well as to the nodal pressure within the saturated domain (cf. Fig. 4 (c)). No mass loss could be detected for the new element. On the contrary, the simulation results predicted by RAM-RTM ${ }^{\circledR}$ show slight deviations (cf. Fig. 4 (c)), which are associated with a mass loss of $0.527 \%$. 
Two-dimensional flow scenario. Additional investigation on a two-dimensional flow scenario also shows a very good agreement between the analytical solution and numerical results (cf. Fig. 5 (a)). A constant pressure of $20 \mathrm{MPa}$ is applied to a central circular injection gate $\left(r_{\text {in }}=4 \mathrm{~mm}\right)$. An identical structured mesh of 6392 Elements was used in Abaqus and PAM-RTM ${ }^{\circledR}$. The flow front propagation is rotationally symmetric, as predicted by the analytical solution for an isotropic permeability tensor (cf. Equation 9) [9]. It has to be noted, that the analytical solution is only valid until the flow front reaches a wall. The mass losses detected during the $2 \mathrm{D}$-verification with sufficient model parameters was below $0.01 \%$.

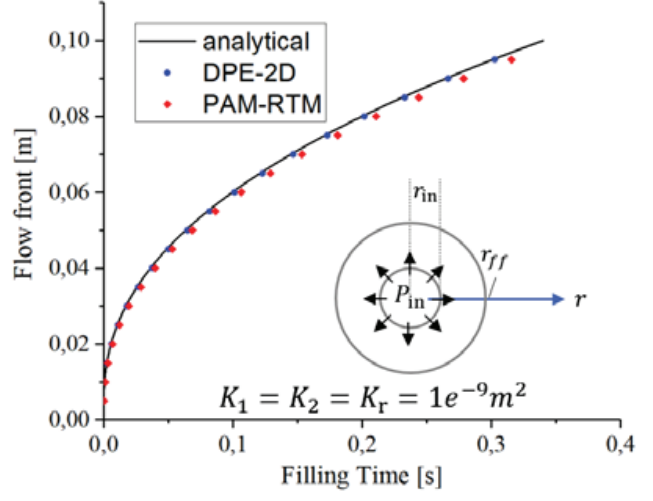

(a)

$$
t_{\mathrm{ff}}=\frac{\eta * \phi}{2 K_{\mathrm{r}} * p_{\mathrm{in}}}\left[r_{\mathrm{ff}}^{2} * \ln \left(\frac{r_{\mathrm{ff}}}{r_{\mathrm{in}}}\right)-\frac{1}{2}\left(r_{\mathrm{ff}}^{2}-r_{\mathrm{in}}^{2}\right)\right]
$$

(b)

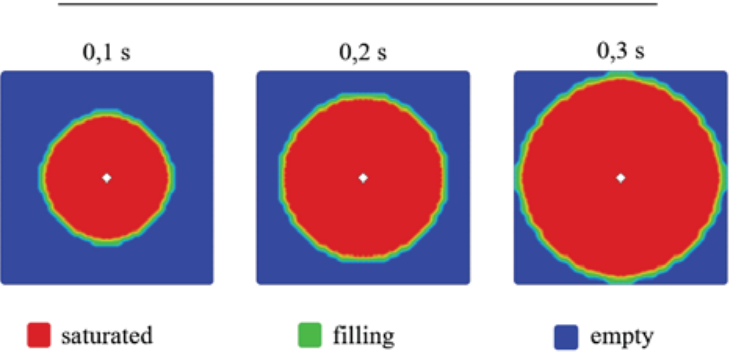

(c)

FIGRUE 5. Verification results for a radial-symmetric two-dimensional flow: (a) Progression of the flow front due to eq. 9; (b) FE/CV-result of the DPE-2D Element for three filling times for a double symmetric mesh containing 6392 Elements.

Superimposed forming scenario. To demonstrate the functionality of the DPE-2D -Element within the proposed framework for FE forming simulation (cf. Fig. 2), a forming simulation with a single ply for a generic geometry (double dome) is superimposed to the mould filling simulation (cf. Fig. 2 (a)). A constant pressure of 1,0 MPa is applied along a central injection line (cf. Fig. 6 (c). The outer edges of the laminate are treated as outlet edges. Hitherto, no thermal contact is applied between the tools and the ply. The flow front propagation during the forming process is shown in Fig. 6 (c) as well as the filling times during the simulation (cf. Fig. 6 (b)).

(a)

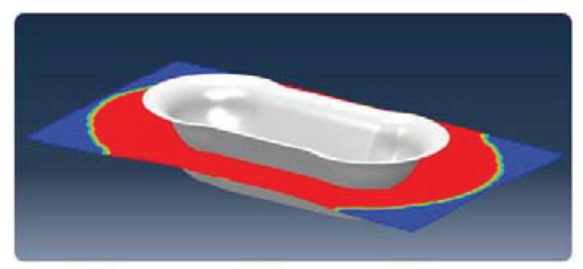

saturated

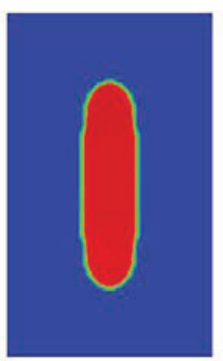

filling

empty

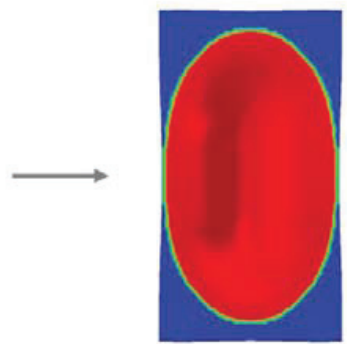

(b)
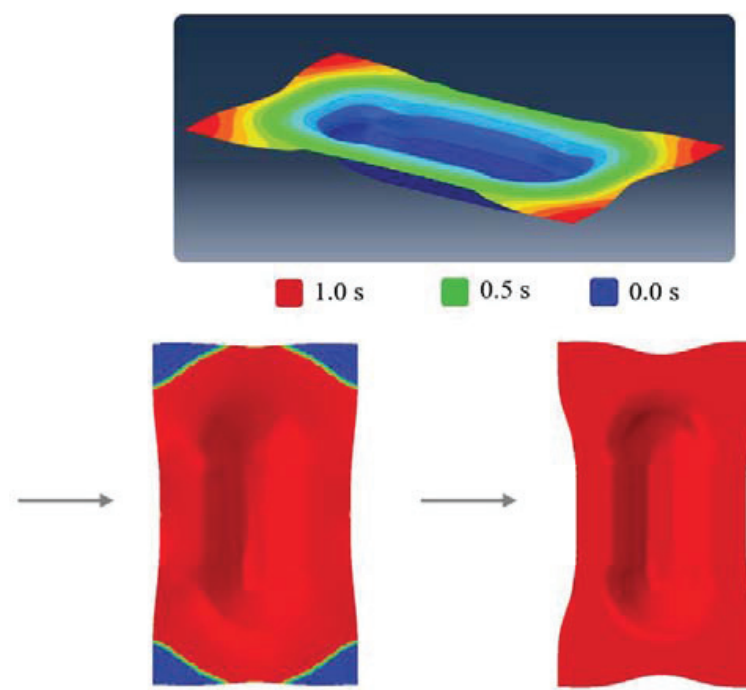

(c)

FIGRUE 6. (a) FE-Forming simulation of a double dome geometry with simultaneous 2D-resin progression using a line injection; (b) Filling times during injection ( $0 \mathrm{~s}$, to $1,0 \mathrm{~s}$ ); (c) flow front progression within the single ply. 


\section{DISCUSSION AND CONCLUSION}

A new two-dimensional element for the modelling macroscopic progression of a single-phase flow front in porous media during FE-forming simulations is presented. The new element is implemented in Abaqus via several user subroutines within an explicit fully coupled thermal-displacement analysis and embedded in the forming simulation approach proposed by Dörr et al. [7,8]. The new approach is verified by comparison with analytical and numerical solutions for one- and two-dimensional test-scenarios. Due to the preconditioned explicit solving algorithm (forming model) a complete incompressibility cannot be modelled. However, sufficient model parameters are identified to ensure convergence against the analytical solution. Mass losses could be reduced to a level even better than state of the art simulation software by implementation of an additional global control volume (CV).

Although no FSI is implemented at the current state, the fibre reorientation during the forming process is directly considered in the fluid propagation element by the formulation of Darcy's law within a fibre parallel material frame, which takes into account arbitrary shear deformations. In addition, the material parameters of the forming model can be modified locally due to the presence and the state of the resin. This enables further investigations of the physical mechanisms regarding the wet compression moulding (WCM) process in terms of the resin's influence on the material parameters and the formability of the infiltrating textiles.

The presented element is a first step in terms of process simulation of the WCM-process because of its twodimensional nature, which disregards any effects in thickness direction such as compaction. Thickness effects, however, are expected to be crucial for an accurate descriptions of the physical mechanisms within the WCM-process [1-3,9]. Therefore, future work will focus on the implementation of a three-dimensional approach for fluid progression in FE-forming simulation. Nonetheless, the presented element can already be used in process simulations where compaction of the laminates is small and where fluid progression is induced by constant pressure or volume flow inlet.

\section{ACKNOWLEDGMENTS}

The authors would like to thank the German State Ministry for Science, Research and Art of Baden-Württemberg (MWK) for the funding of the project Forschungsbrücke Karlsruhe-Stuttgart for which the presented work is carried out. Additionally, the authors would like to thank their project partners at Stuttgart University, Institute for Aircraft Design (IFB) under leadership of Prof. Middendorf for the collaboration. The work is part of the Young Investigator Group (YIG) “Tailored Composite Materials for Lightweight Vehicles”, generously funded by the Vector Stiftung.

\section{REFERENCES}

1. J. Bergmann, H. Dörmann, R. Lange, "Interpreting process data of wet pressing process. Part 1: Theoretical approach”, Journal of Composite Materials, Vol. 50, pp. 2399-2407 (2016).

2. J. Bergmann, H. Dörmann, R. Lange, "Interpreting process data of wet pressing process. Part 2: Verification with real values", Journal of Composite Materials, Vol. 50, pp. 2409-2419 (2016).

3. K. Heudorfer, C. Carosella, P. Middendorf, "Compression wet moulding as alternative to RTM", Conference proceedings $25^{\text {th }}$ for Stuttgarter Kunststoffkolloquium (22-23.03.2017, Stuttgart).

4. F. Trochu, R. Gauvin, D. Gao, "Numerical Analysis of the Resin Transfer Molding Process by the Finite Element Method", Advances in Polymer Technology, Vol. 12, No. 4, pp. 329-342 (1993).

5. M. Buschke, S. Advani, "A Finite Element/Control Volume Approach to Mold Filling in Anisotropic Porous Media”, Polymer Composites, Vol. 11, pp. 398-405 (1990).

6. S.C. Joshi, Y.C. Lam, X.-L. Liu "Mass conservation in numerical simulation of resin flow", Composites Part A (Vol. 31, pp. 1061-1068, 2000), DOI: 10.1016/S1359-835X(00)00067-1

7. D. Dörr, T. Joppich, F. Schirmaier, T. Mosthaf, L. Kärger, F. Henning, "A method for validation of finite element forming simulation on basis of a pointwise comparison of distance and curvature", AIP Conference Proceedings 1769170011 ESAFROM 2016, Nantes France, DOI: 10.1063/1.4963567 (2016).

8. D. Dörr, F. Schirmaier, F. Henning, L. Kärger, "A viscoelastic approach for modeling bending behavior in finite element forming simulation of continuously fiber reinforced composites”, Composites Part A, Vol. 94, pp. 113123 (2017).

9. C. D. Rudd, A. C. Long, K. N. Kendall, C. G. M. Mangin, "Liquid Moulding Technologies - Resin transfer moulding, structural reaction injection moulding and related processing techniques", ISBN 978-1-84569-544-6 (online), Woodhead Publishing Limited. (1997). 